\title{
Time-to-Target Simplifies Optimal Control of Visuomotor Feedback Responses
}

\author{
Justinas Česonis and $\oplus^{D}$ David W. Franklin
}

https://doi.org/10.1523/ENEURO.0514-19.2020

Department of Sport and Health Sciences, Technical University of Munich, 80992 Munich, Germany

\begin{abstract}
Visuomotor feedback responses vary in intensity throughout a reach, commonly explained by optimal control. Here, we show that the optimal control for a range of movements with the same goal can be simplified to a time-to-target dependent control scheme. We measure our human participants' visuomotor responses in five reaching conditions, each with different hand or cursor kinematics. Participants only produced different feedback responses when these kinematic changes resulted in different times-to-target. We complement our experimental data with a range of finite and non-finite horizon optimal feedback control (OFC) models, finding that the model with time-to-target as one of the input parameters best replicates the experimental data. Overall, this suggests that time-to-target is a critical control parameter in online feedback control. Moreover, we propose that for a specific task and known dynamics, humans can instantly produce a control signal without any additional online computation allowing rapid response onset and close to optimal control.
\end{abstract}

Key words: motor control; optimal feedback control; reaching; time-to-target; visuomotor control; visuomotor feedback response

\section{Significance Statement}

Human behavior has widely been explained using stochastic optimal feedback control (OFC), formulating movement control as a set of time-dependent feedback and control gains. However, OFC is computationally expensive leading to questions about whether such a theory could be implemented in real time. Here, we show that OFC could be approximated by a simple relationship between feedback gains and the time-totarget over a variety of movement kinematics, matching the evolution of visuomotor feedback gains of our human participants during reaching. As this relationship to time-to-target is similar across a wide range of kinematics, this suggests that early stages of the OFC controlled movement could be approximated by a time-to-target control, saving computational costs and allowing for rapid execution.

\section{Introduction}

From intercepting a basketball pass between opponents to catching a vase accidentally knocked off the shelf, visuomotor feedback responses play a familiar role in human motor behavior. Previous research has extensively analyzed

\footnotetext{
Received December 9, 2019; accepted March 1, 2020; First published March 20, 2020.

The authors declare no competing financial interests.

Author contributions: J.Č. and D.W.F. designed research; J.Č. and D.W.F. performed research; D.W.F. contributed unpublished reagents/analytic tools; J.Č. analyzed data; J.Č. and D.W.F. wrote the paper.

Acknowledgements: We thank Matthew Millard, Michael Dimitriou, Sae Franklin, Raz Leib, and Marion Forano for their comments on an earlier version of this manuscript.
}

these responses in human reaching movements (Day and Lyon, 2000; Saunders and Knill, 2003, 2004, 2005; Sarlegna et al., 2003; Knill et al., 2011; Reichenbach et al., 2014; de Brouwer et al., 2017, 2018), and showed an interesting combination of task-dependent variability on the timescale of a single movement (Dimitriou et al.,

Correspondence should be addressed to David W. Franklin at david. franklin@tum.de.

https://doi.org/10.1523/ENEURO.0514-19.2020

Copyright () 2020 Česonis and Franklin

This is an open-access article distributed under the terms of the Creative Commons Attribution 4.0 International license, which permits unrestricted use, distribution and reproduction in any medium provided that the original work is properly attributed. 
2013; Franklin et al., 2014, 2017; Cross et al., 2019), as well as sub-voluntary feedback onset times (Prablanc and Martin, 1992; Day and Lyon, 2000; Franklin and Wolpert, 2008; Oostwoud Wijdenes et al., 2011; Zhang et al., 2018). These visuomotor feedback responses have been shown to modulate throughout a movement depending on the perturbation onset location (Dimitriou et al., 2013). This observation was explained through optimality principles, however such control was modeled only indirectly, by replicating velocity profiles and trajectories of visually perturbed movements (Liu and Todorov, 2007; Rigoux and Guigon, 2012). In this study, we test to what degree optimal feedback control (OFC) can be used to model the visuomotor feedback responses directly.

Optimal control as a theory of human movement has normally been compared against other theories in terms of prediction of kinematics and dynamics (Todorov and Jordan, 2002; Izawa et al., 2008; Guigon et al., 2007, 2008; Nagengast et al., 2009; Yeo et al., 2016). Nevertheless, OFC has been used to motivate extensive studies investigating the control and task-dependent modulation of feedback responses (Knill et al., 2011; Pruszynski and Scott, 2012; Nashed et al., 2012, 2014). The results of these and other studies have highlighted the flexibility of the modulation of these feedback responses. While a few studies have compared the predictions of the controller feedback gains against the feedback responses in human subjects (Knill et al., 2011), such predictions have not been made about the temporal evolution of these feedback responses during reaching. For example, Dimitriou et al., 2013) show temporal evolution of feedback response intensity throughout a reaching movement, suggesting that this is similar to the feedback gain predictions of Liu and Todorov (2007). However, a direct comparison of these feedback intensities has not been made. Here, we directly compare the temporal evolution of visuomotor feedback response intensities in human participants with the prediction of these intensities in an OFC model.

Visuomotor feedback response intensity over a goal directed reaching movement follows a roughly bell-shaped profile, with peak intensity in the middle and decay toward the beginning and the end of the movement (Dimitriou et al., 2013). The results of Liu and Todorov (2007) suggest that such modulation is a combination of gains related to movement position, velocity and acceleration. However, we do not yet know whether these gains would more strongly depend on the visual kinematics or haptic kinematics. In addition, models of ball catching were shown to produce systematic errors in the prediction of the hand kinematics when using only velocity or acceleration based gains (Dessing et al., 2002), suggesting an integration of multiple state variables to produce the feedback response. Evidence of such integration then raises two important questions. First, could there be other states than position and its derivatives that also contribute to such control? Second, how can these responses be produced so rapidly, when multiple inputs need to be integrated into one solution?
One method to solve these two problems would be a controller based on time-to-target. Within a state-space system, all state variables are constantly changing with time with a fixed relationship to one another as described by the state transition and control matrices. Such a system can then be re-imagined as a system with time as its input, and these physical states as the hidden states. Such mapping simplifies the multiple input system where the inputs are state variables, to a one-input (time) system. Indeed, the expected time-to-target (or time-tocontact) has been shown to be related to the control in finger pointing (Oostwoud Wijdenes et al., 2011) and catching tasks (Dessing et al., 2002). Therefore, we test whether a simple relation to the time-to-target can explain the temporal profile of visuomotor feedback responses in humans. To test our hypotheses, we devised an experimental paradigm where we offset the usual bell-shaped velocity profile in the aim to separate the effect of the times-to-target from the effect of kinematics (both, of the hand and of the cursor) on the visuomotor feedback responses. Finally, we compare these results with a normative OFC model of visuomotor feedback responses to better understand how and whether these responses can be the result of optimality and still maintain rapid onset times.

\section{Materials and Methods}

\section{Code availability}

The code and the experimental data described in the paper is freely available online at https://doi.org/ $10.6084 / \mathrm{m} 9$.figshare.11323289. The code is available as Extended Data 1.

\section{Participants}

Eleven right-handed (Oldfield, 1971) human participants (five females; $27.3 \pm 4.5$ years of age) with no known neurologic diseases took part in the experiment. All participants provided written informed consent before participating. All participants except one were naive to the purpose of the study. Each participant took part in five separate experimental sessions, each of which took $\sim 3 \mathrm{~h}$. One participant was removed from analysis as their kinematic profiles under the five experimental sessions overlapped. The study was approved by the Ethics Committee of the Medical Faculty of the Technical University of Munich.

\section{Experimental setup}

Participants performed forward reaching movements to a target while grasping the handle of a robotic manipulandum with their right hand. Participants were seated in an adjustable chair and restrained using a four-point harness. The right arm of participants was supported on an air sled while grasping the handle of a planar robotic interface (vBOT; Howard et al., 2009). A six-axis force transducer (ATI Nano 25; ATI Industrial Automation) measured the end-point forces applied by the participant on the handle. Position and force data were sampled at $1 \mathrm{kHz}$. Visual feedback was provided in the plane of the hand via 

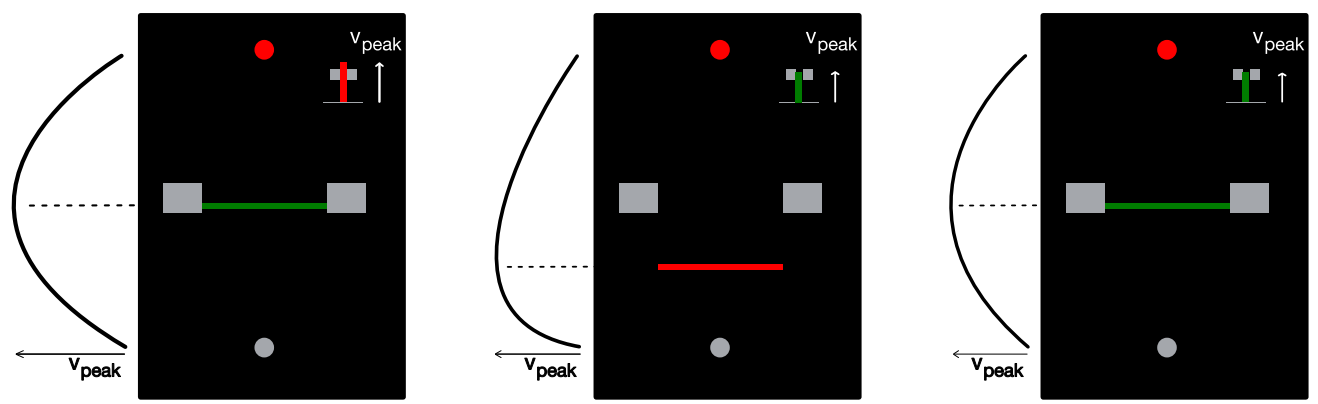

Figure 1. Examples of feedback presented to the participants. Feedback regarding the peak velocity and the timing of the peak velocity was provided after each trial. Large gray blocks indicate the velocity peak location target, while the bar chart at the top-right corner indicates peak $y$-velocity magnitude. Feedback was provided on the modality (cursor or hand) that matched the baseline, where the horizontal line indicated the location of the peak velocity in this modality. Left, Velocity peak location is within the target, but the movement was too fast (unsuccessful trial). Middle, Velocity peak location is too early, but the movement speed is within the target (unsuccessful trial). Right, Successful trial.

a computer monitor and a mirror system, such that this system prevented direct visual feedback of the hand and arm. The exact onset time of any visual stimulus presented to the participant was determined from the graphics card refresh signal.

Participants initiated each trial by moving the cursor (yellow circle of $1.0 \mathrm{~cm}$ diameter) into the start position (gray circle of $1.6 \mathrm{~cm}$ diameter) located $\sim 25 \mathrm{~cm}$ in front of the participant, centered with their body. This start position turned from gray to white once the cursor was within the start position. Once the hand was within the start position for a random delay drawn from a truncated exponential distribution (1.0-2.0 s, mean $1.43 \mathrm{~s}$ ), a go cue (short beep) was provided signaling participants to initiate a straight reaching movement to the target (red circle of $1.2 \mathrm{~cm}$ diameter, located $25.0 \mathrm{~cm}$ directly in front of the start position). If participants failed to initiate the movement within $1000 \mathrm{~ms}$, the trial was aborted and restarted. Once the cursor was within $0.6 \mathrm{~cm}$ of the center of the target, participants were notified by the target changing color to white. The movement was considered complete when the participants maintained the cursor continuously within this $0.6 \mathrm{~cm}$ region for $600 \mathrm{~ms}$. If participants did not complete the movement within $4 \mathrm{~s}$ from first arriving at the start position (e.g., by undershooting or overshooting the target), the movement timed-out and had to be repeated. Otherwise, as long as participants arrived at the target within $4 \mathrm{~s}$, the trial was considered to have been completed. After each trial, the participant's hand was passively returned by the robot to the start position while visual feedback regarding the success of the previous trial was provided (Fig. 1). Movements were self-paced, and short breaks were enforced after every 100 trials.

\section{Experimental paradigm}

Participants performed the experiment under five different conditions, each performed in a separate session. In the baseline condition the cursor matched the forward movement of the hand, with a peak velocity in the middle of the movement. In the other four conditions, the cursor location was scaled relative to the hand location in the forward direction only (with no change in the lateral direction), such that the cursor and the hand location matched only at the start and end of the movements (Fig. 2). In two of the conditions (matched-hand velocity), the hand velocity matched the baseline condition throughout the movement (with the peak in the middle of the movement) but the cursor velocity peaked either earlier $(33 \%$ of movement distance) or later (66\% of movement distance). In the other two conditions (matched-cursor velocity), the cursor velocity was matched to the baseline condition throughout the movement (with the peak in the middle of the movement) but the hand velocity peaked either earlier (33\% of movement distance) or later (66\% of movement distance). The difference between the cursor velocity and the hand velocity was produced through a linear scaling of the cursor velocity as a function of the forward position (Fig. 2A). Specifically, for the two conditions where the position of the peak cursor velocity is earlier than the position of the peak hand velocity (Fig. 2, top), this scaling was implemented as:

$$
\frac{v_{c}}{v_{h}}=-0.012 d+1.6
$$

where $v_{c}$ and $v_{h}$ are cursor and hand velocities, respectively, and $d$ is the distance along the movement direction in $\%$. The cursor velocity was therefore manipulated by a linear scaling function such that its velocity is $160 \%$ of the hand velocity at the beginning of the movement, linearly decreasing to $40 \%$ at the target location (Fig. 2, top). For the two conditions where the position of the peak cursor velocity is later than the position of the peak hand velocity (Fig. 2, bottom), this scaling was implemented as:

$$
\frac{v_{c}}{v_{h}}=0.012 d+0.4,
$$

such that the velocity gain function linearly increased from $40 \%$ hand velocity at the start of the movement to $160 \%$ at the end of the movement (Fig. 2, bottom). Desired velocity profiles of both the hand and the cursor are shown in Figure 2B for each condition.

Introducing the differences in velocity profiles across five experimental conditions allows us to look at the effect of the time-to-target separately from the kinematics of 


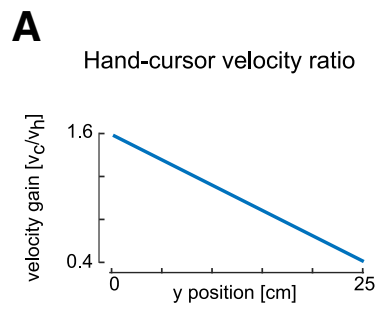

B

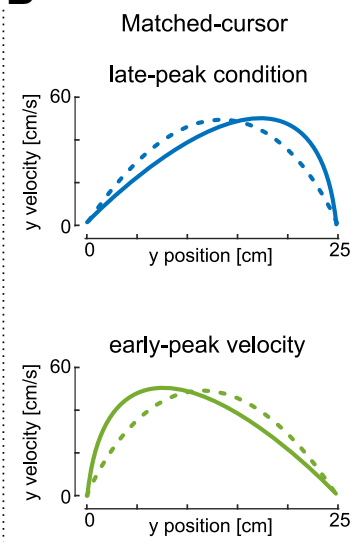

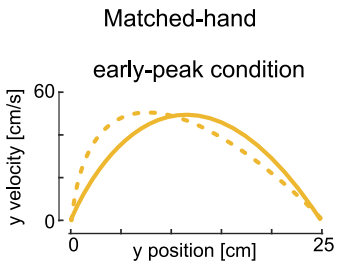

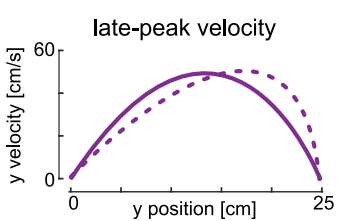

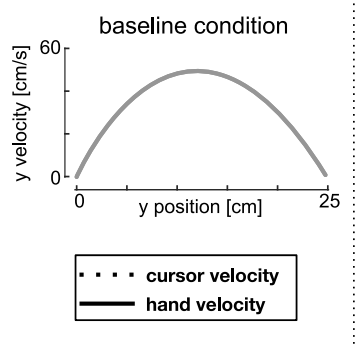

Figure 2. Experimental design. $\boldsymbol{A}$, top, Hand-cursor velocity scaling for conditions where the cursor position leads the hand position in $y$-axis (matched-cursor late-peak hand velocity condition, blue, and matched-hand early-peak cursor velocity condition, yellow). Bottom, Hand-cursor velocity scaling for conditions where the cursor position lags the hand position in $y$-axis (matched-cursor early-peak hand velocity condition, green, and matched-hand late-peak cursor velocity condition, purple). B, Hand and cursor velocity-position profiles required to achieve the ideal movement to the target. Left, Matched-cursor velocity conditions. Middle, Baseline condition, where cursor position and hand position are consistent. Right, Matched-hand velocity conditions.

physical movement. First, as the visual perturbations always occurred at the five preset hand positions, our design allows us to maintain the effect of the distance to the target and distance in the movement constant across all five conditions. Second, two perturbation locations (onethird and two-thirds of movement distance) were chosen so that velocities at those locations matched across multiple conditions (early-peak condition velocity equals baseline at one-third, and late-peak velocity equals baseline at two-thirds), allowing for matching velocity contributions across conditions as well. However, across the three different physical kinematics the time-to-target is varied, allowing us to investigate whether time-to-target has an effect. Finally, the relative scaling between cursor velocity and hand velocity in the forward direction also separates the relative contributions of these two inputs, allowing us to examine the relative contributions of visual and physical kinematics in modulating the feedback responses.

\section{Feedback regarding movement kinematics}

In all conditions, one of the velocity modalities (cursor or hand) was required to be similar to the baseline velocity profile. Feedback was always provided about the velocity modality that matched the baseline. Ideal trials were defined as trials in which this peak velocity was between 42 and $58 \mathrm{~cm} / \mathrm{s}$ with the peak location between $45 \%$ and $55 \%$ of the movement distance with no target overshoot. Participants were credited one point for achieving an ideal trial and zero points otherwise, however all the trials were included in the analysis. After each trial, visual feedback about the peak velocity and the location at which this peak occurred was provided to the participants graphically (Fig. 1). The peak velocity was indicated on the righthand side of the screen with the length of a bar and the velocity target. This bar changed color from red to green if the velocity was within the ideal range. The location of the peak velocity was indicated as a horizontal line between home and target positions at the exact location it was achieved, along with the ideal range. This line was green when the location of the peak velocity was within the ideal range, and red otherwise. Overshooting the target was defined as the position of the cursor exceeding the center of the target in the forward direction by $>0.9 \mathrm{~cm}$. If participants reached the target while overshooting during the movement, a message indicating the overshot was shown, no points were scored and an error tone was played to discourage further overshooting movements.

\section{Probe trials}

During each session, probe trials were used to measure the visuomotor feedback intensity, the average strength of corrective motor response to a change in the visual feedback of hand position. To elicit these feedback responses (further visuomotor feedback responses), visual perturbations were initiated laterally $( \pm 2.0 \mathrm{~cm})$ at five different hand distances $(4.2,8.3,12.5,16.7$, and $20.8 \mathrm{~cm})$ from the start (Fig. $3 A$ ). In addition, a zero-amplitude perturbation (cursor matched to the lateral position of the hand) was included, resulting in eleven different probe trials. On these trials the visual perturbations lasted $250 \mathrm{~ms}$, after which the cursor was returned to the lateral location of the hand. The lateral hand position was constrained in these trials in a simulated mechanical channel throughout the movement, thereby requiring no correction to reach the target. The simulated mechanical channel was implemented with a stiffness of $4000 \mathrm{~N} / \mathrm{m}$ and damping of 2 $\mathrm{Ns} / \mathrm{m}$ acting perpendicularly to the line connecting the start position and the target (Scheidt et al., 2000; Milner and Franklin, 2005), allowing measurement of any lateral forces in response to a visual perturbation.

In previous experiments, feedback response intensity gradually decreased during the course of the experiment (Franklin and Wolpert, 2008; Franklin et al., 2012). However, it has been shown that including perturbation trials where 
A

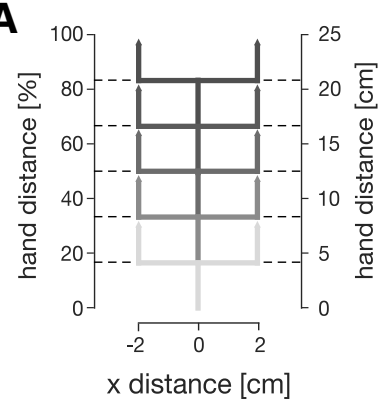

B

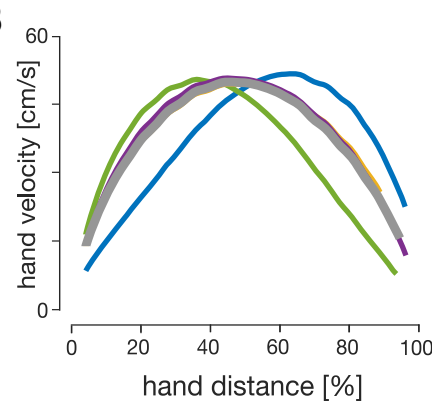

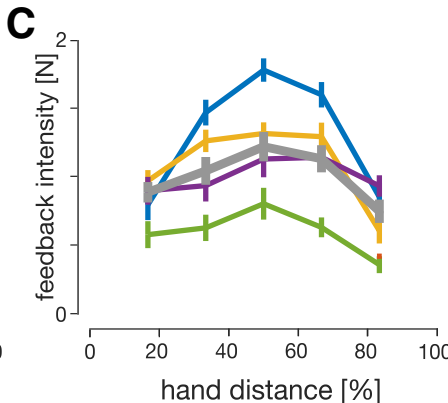

Figure 3. Human visuomotor feedback responses are modulated across the five experimental conditions. $\boldsymbol{A}$, Lateral perturbations of the cursor were applied in all five conditions. Perturbations were introduced as 2-cm cursor jumps perpendicular to the movement direction. The perturbation onset occurred at one of five equally spaced hand locations. $\boldsymbol{B}$, Mean velocity profiles of the hand in five experimental conditions: matched-cursor early-peak (green), matched-cursor late-peak (blue), matched-hand early-peak (yellow), matched-hand late-peak (purple), and baseline (gray). Participants successfully modulated forward movement kinematics to meet task demands, velocity profiles are skewed for matched-cursor conditions, and are similar to the baseline for matched-hand conditions. C, Mean visuomotor feedback intensities (mean lateral force from 180 to $230 \mathrm{~ms}$ after perturbation onset) across all participants to cursor perturbations as a function of the hand distance in the movement. Error bars represent 1 standard error of the mean (SEM). Significant regulation is observed for matched-cursor early-peak and matched-cursor late-peak conditions (blue and green), but no significant regulation is seen for matched-hand conditions (yellow and purple), relative to the baseline.

the perturbations were maintained until the end of the movement, and where participants had to actively correct for the perturbation to reach the target, prevents this decrease in the feedback intensity (Franklin et al., 2016). Therefore, half of the trials contained the same range of perturbations as the probe trials but where these perturbations were maintained throughout the rest of the trial and participants had to correct for this perturbation. The hand movement was not constrained in these maintained perturbation trials. These maintained perturbations have now been used in several studies (Franklin et al., 2016, 2017; de Brouwer et al., 2017).

\section{Session design}

Before each session, participants performed 100-300 training trials to learn the specific velocity profiles of the reaching movements. All training trials contained no visual perturbations and were performed in the null force field. The training trials were stopped early once participants achieved an accuracy of $75 \%$ over the last 20 trials and were not used for the analysis.

Each session consisted of 40 blocks, where each block consisted of 22 trials performed in a randomized order. Eleven of these 22 trials were probe trials (five perturbation locations $\times$ two perturbation directions + zero perturbation condition) performed in the mechanical channel. The other eleven trials consisted of the same perturbations but maintained throughout the trial and performed in the null field. Therefore, in each of the five sessions, participants performed a total 880 trials (440 probe trials). The order of the five different conditions (sessions) was pseudo-randomized and counterbalanced across participants. Participants were not told about the physical implementation of the different mappings, but were provided feedback after every trial and knew that each session was different from previous sessions.

\section{Data analysis}

Data were analyzed in MATLAB R2017b and JASP 0.8 .2 . Force and kinematic time series were low-pass filtered with a tenth-order zero-phase-lag Butterworth filter $(40-\mathrm{Hz}$ cutoff). The cursor velocity was calculated by multiplying the hand velocity by the appropriate scaling function. The visuomotor feedback response was measured for each perturbation location as the difference between the force responses to the leftward and rightward perturbations within a block. To measure the visuomotor feedback response intensity (mean force, produced as a response to a fixed-size visual perturbation) this response was averaged over a time window of $180-230 \mathrm{~ms}$, a commonly used time interval for the involuntary visuomotor feedback response (Franklin and Wolpert, 2008; Franklin et al., 2012, 2016; Dimitriou et al., 2013). In order to compare any differences across the conditions a two-way repeated-measures ANOVA was performed with main effects of condition (five levels) and perturbation location (five levels). As a secondary method to frequentist analysis we also used the Bayesian factor analysis (Raftery and Kass, 1995) to verify our statistical results. Bayesian factor analysis is a method that in addition to the conventional hypothesis testing (evaluating evidence in favor of the alternative hypothesis) allows us to evaluate evidence in favor of the null hypothesis, therefore distinguishing between the rejection of the alternative hypothesis and not enough evidence to accept the alternative hypothesis.

Although we used the time window of $180-230 \mathrm{~ms}$ to estimate visuomotor feedback intensity, we also verified whether the onset of the visuomotor feedback response in our data are consistent with previously reported values. To estimate this onset time, we first estimated individual onset times for each participant at each perturbation location and movement condition. To do so, we used the receiver operator characteristic (ROC) to estimate where the force reaction to leftwards cursor perturbations deviated from the reaction to rightwards cursor perturbations (Pruszynski et al., 2008). For each type of trials, we built the ROC curve for the two signals at $1 \mathrm{~ms}$ intervals, starting from $50 \mathrm{~ms}$ before the perturbation, and calculated the area under this curve (aROC) for each of these points 
until the aROC exceeded 0.75 for 10 consecutive milliseconds. In order to find where the force traces start deviating from each other, we then fit a function of the form $\max (0.5, k \times(t-\tau)$ to the aROC curve. The time point where the linear component of this function first overtakes the constant component was taken as the threshold value. Overall, the mean onset times across all conditions and perturbation locations were $138 \pm 7 \mathrm{~ms}$ (mean $\pm \mathrm{SD}$ ), with onset times consistent among movement conditions $\left(F_{(4,36)}=1.410, p=0.25\right.$, and $\left.B F_{10}=0.105\right)$, perturbation locations $\left(F_{(4,36)}=1.582, p=0.20, B F_{10}=0.252\right)$, and their interactions $\left(F_{(16,144)}=1.350, p=0.176\right.$, and $\left.B F_{10}=0.005\right)$

\section{Modeling \\ OFC}

In addition to our linear models we implemented two different OFC models: the classical model (Liu and Todorov, 2007) and the time-to-target model. The only comparison between the output of the optimal control models and the experimental results is via the feedback gains. For each movement we define time-to-target as the duration between the onset of the perturbation and the cursor first intercepting the target. In both models we modeled the hand as a point mass of $m=1.1 \mathrm{~kg}$ and the intrinsic muscle damping as a viscosity $b=7 \mathrm{Ns} / \mathrm{m}$. This point mass was controlled in a horizontal plane by two orthogonal force actuators to simulate muscles. These actuators were controlled by the control signal $u_{t}$ via a first order low-pass filter with a time constant $\tau=0.05 \mathrm{~s}$. The state-space representation of the dynamic system used to simulate the reaching movements can be expressed as

$$
x_{t+1}=A x_{t}+B(I+C) u_{t}+\xi_{t},
$$

where $A$ is a state transition matrix, $B$ is a control matrix, and $C$ is a $2 \times 2$ matrix, whose each element is a zeromean normal distribution representing control-dependent noise. Variables $x_{t}$ and $u_{t}$ are state and control at time $t$, respectively. State $x_{t}$ exists in the Cartesian plane and consists of position $\vec{p}$ (2 dimensions), velocity $\vec{v}$ (2), force $\vec{f}$ (2), and target position $\overrightarrow{p^{*}}$ (2). The presence of these four states within the state vector means that the information about all of these states is eventually used for the control. For our simulation purposes we treat the control-independent noise $\xi_{t}$ as zero.

The state of the plant is not directly observable, but has to be estimated from noisy sensory information. We model the observer as:

$$
y_{t}=H x_{t}+D_{t},
$$

where $H=\operatorname{diag}[1,1,1,1,1,1,0,0]$ is the observation matrix, and $D_{t}$ is a diagonal matrix of zero-mean normal distributions representing state-independent observation noise. Therefore, our observer can infer the state information of position, velocity and applied force of the plant, consistent with human participants.

The simulated movements were guided by the LQG controller with a state-dependent cost $\mathrm{Q}$, an activation cost $\mathrm{R}$, a reaching time $\mathrm{N}$, and a time step $t=0.01 \mathrm{~s}$. However, due to the presence of the control-dependent noise, the estimation and control processes are not anymore separable as in the classic LQG theory. In order to obtain optimal control and Kalman gain matrices we used the algorithm proposed by Todorov and Li (2005), where control and Kalman gain matrices are iteratively updated until convergence.

For both the classical and time-to-target models we simulated three different movement kinematics representing three different conditions in our experiment, the baseline and the two matched-cursor conditions. The state-dependent $\operatorname{cost} Q$ was identical for all three kinematics:

$$
\begin{aligned}
& Q(t) \\
& =\left\{\begin{array}{l}
0, \text { for } t \neq N \\
\left(\overrightarrow{\omega_{p}}\left(\vec{p}(t)-\overrightarrow{p^{*}}(t)\right)\right)^{2}+\omega_{v}\|\vec{v}(t)\|^{2}+\omega_{f}|| \vec{f}(t) \|^{2}, \text { for } t=N
\end{array}\right.
\end{aligned}
$$

where $\overrightarrow{\omega_{p}}=[0.5,1], \omega_{v}=0.02$, and $\omega_{f}=2$. The activation cost $R(t)=0.00001$ was constant throughout the movement for the baseline condition, but was modulated for the two matched-cursor conditions by multiplying it elementwise by a scaling function:

$$
R^{\prime}(t)=\frac{\exp \left(p \frac{t+q}{r}\right)}{\operatorname{mean}\left(R^{\prime}\right)},
$$

where $p, q$, and $r$ are constants.

Thus, each movement condition only differed from the other two by the profile of this activation cost $R$, but not by its magnitude. These modified activation costs shift the timing of the peak velocity toward either the beginning or the end of the movement by penalizing higher activations at either the end or beginning of the movements, respectively.

The mean activation cost is kept constant across the conditions resulting in each condition being equally "effortful." All other simulation parameters were kept constant across the three conditions.

Although LQG is a fixed time horizon problem, we did not predefine the movement duration $\mathrm{N}$. Instead, we obtained the $\mathrm{N}$, and constants $p, q$, and $r$ using Bayesian adaptive direct search (BADS; Acerbi and Ma, 2017) to maximize the log-likelihood of the desired peak velocity location and magnitude. We did not fit any other parameters beyond this point. Rather, we analyzed our models' qualitative behavior compared with human participant data.

The classical and the time-to-target models only differed in the way the perturbations were handled. For the classical model, we simulated perturbation trials at every time step $t_{p}$ by shifting the target $x$-coordinate by $2 \mathrm{~cm}$ at the time $t_{p}+120 \mathrm{~ms}$. This $120 \mathrm{~ms}$ delay was used to mimic the visuomotor delay in human participants, and was taken from Liu and Todorov (2007). We then averaged the force response of the controller over the time window $\left[t_{p}+130\right.$, $\left.t_{p}+180\right]$ as an estimate of the simulated feedback responses, equivalent of visuomotor feedback responses in our participants. This means that our simulated feedback responses arise due to separate contributions from the controller position, velocity and acceleration gains. For perturbations occurring at times where the movement is over 
before the end of this time window, the intensity of this simulated feedback response is set to zero.

For the time-to-target model we introduced an extension in the time-to-target after the onset of any perturbation similar to that observed in our participants. Simulated feedback intensities were modeled at five locations, matching the perturbation locations in our experiment to obtain the appropriate increase in time-to-target after each perturbation. In order to simulate the response to perturbations we first extracted the perturbation onset times from movement kinematics by performing an unperturbed movement and recording the time point $t_{p}$ at which this movement passed the perturbation onset location. We then simulated the postperturbation portion of the movement as a new LQG movement with an initial state matching the state at $t_{p}+120 \mathrm{~ms}$ of the unperturbed movement, and movement duration matching the time-to-target recorded in our participants for the particular perturbation. Therefore, our time-to-target model can only simulate the feedback intensities at the five perturbation locations in the movement. Together, this keeps our simulated reaches "naive" to the perturbation before its onset and allows the time-to-target of the simulated reaches to match the respective time-to-target of our human participants. Finally, we calculated the simulated feedback intensities as described previously, using a time window [10, $60 \mathrm{~ms}]$ of the postperturbation movement. As in the previous simulations, these simulated feedback responses arise due to separate contributions from the controller position, velocity and acceleration gains.

\section{Time-to-target tuning function}

In order to understand the mechanisms that might underlie the consistent relationship between the simulated feedback intensities and the time-to-target, we fit a mathematical expression to the simulated feedback intensities. We modeled the relationship as the minimum of a squared-hyperbolic function and a logistic function:

$$
G(t)=\min \left(\frac{\beta}{\left(t-t_{1}\right)^{2}} ; \frac{\alpha}{1+\exp \left(-\frac{t-t_{0}}{\tau}\right)}\right),
$$

and used BADS to fit this function to our time-to-target-simulated feedback intensity data by optimizing the loglikelihood of this fit.

While the logistic function was chosen simply as it provided a good fit to the data, the squared-hyperbolic arises from the physics of the system. Specifically, from the kinematic equations of motion for a point mass $(m)$ traveling a distance $(d)$ under the influence of force $F$, the distance can be expressed as:

$$
d=\frac{F t^{2}}{2 m}+v_{0} t
$$

where $v_{0}=0$ is the lateral velocity at the start of perturbation correction. Rearranging gives:

$$
F=\frac{2 m d}{t^{2}} \propto \frac{1}{t^{2}} .
$$

Hence the lateral force necessary to bring a point mass to the target is proportional to $1 / t^{2}$.

\section{Receding horizon OFC}

In addition to our finite horizon control we also implemented a receding horizon controller (Guigon et al., 2019). Irrespectively of the current state of the movement $X_{t}$, the receding horizon controller is defined to aim to arrive at the target at time $t+T_{h}$. In essence, such controller is therefore not different from the finite horizon controller in its implementation for a single state of the movement. We implemented the receding horizon controller by iterating a finite horizon controller described previously, but with the $T_{h}=500 \mathrm{~ms}$, and $\mathrm{Q}$ and $\mathrm{R}$ costs scaled from the finite horizon model to fit the movement duration. For each iteration we recorded the next movement state (10 ms away from the initial state), and used that as the initial state for the next iteration. This process was repeated until the cursor was within the distance of $0.4 \mathrm{~cm}$ from the target position, and remained there without overshooting for $600 \mathrm{~ms}$.

Simulating differently skewed velocity profiles within the framework of receding-horizon control is non-trivial. As a result, we chose to only model one, the baseline, experimental condition, where the activation cost $\mathrm{R}$ is constant within the movement. Therefore, we chose the costs

$$
\begin{gathered}
\quad Q(t) \\
=\left\{\begin{array}{l}
0, \text { for } t \neq T_{h} \\
\overrightarrow{\omega_{p}}\left(\vec{p}(t)-\overrightarrow{p^{*}}(t)\right)^{2}+\omega_{v}\|\vec{v}(t)\|^{2}+\omega_{f}\|\vec{f}(t)\|^{2}, \text { for } t=T_{h},
\end{array}\right.
\end{gathered}
$$

where $\overrightarrow{\omega_{p}}=[5,5], \omega_{v}=0.05$, and $\omega_{f}=5$. and the activation cost $R=0.000003$. The values were selected so that the movement durations, produced by the receding-horizon model would match the experimental durations for the baseline condition. However, the resultant velocity profiles of this model more closely resembled those of the early-peak velocity condition, than those of the baseline. To account for any effects of the velocity profile we also fit the costs so the model prediction of movement durations matched the durations of the earlypeak velocity condition. For this simulation we selected $\overrightarrow{\omega_{p}}=[0.7,0.7], \omega_{v}=0.007$, and $\omega_{f}=0.7$, while the activation cost remained unchanged.

In this model we introduced the simulated perturbation by shifting the target position by $2 \mathrm{~cm}$ at $120 \mathrm{~ms}$ after the $y$-coordinate of the movement passed the perturbation onset location. We only simulated the perturbations matching our experimental conditions, lateral $2 \mathrm{~cm}$ cursor jumps, with the onset at five evenly distributed forward distances. We calculated simulated feedback intensities the same way as for the classical and time-to-target models.

\section{Infinite horizon OFC}

We implemented the infinite horizon OFC to control our simulated hand based on the previous work of Qian et al. (2013). Specifically, we calculated the control gain matrix $\mathrm{L}$, and Kalman gain matrix $\mathrm{K}$ to control the same system as in the previous models. We chose the state-dependent costs $\overrightarrow{\omega_{p}}=[1,1], \omega_{v}=0.02$, and $\omega_{f}=0$ for the baseline condition simulation, and $\overrightarrow{\omega_{p}}=[0.35,0.35], \omega_{v}=0.007$, 
and $\omega_{f}=0$ for the early-peak condition simulation. For both conditions, the activation cost $R=0.002$ was kept the same. The protocol of simulating the mean trajectories, feedback responses and their intensities was otherwise identical to the receding horizon simulations.

\section{Model comparison}

We compared the simulated feedback intensities from each of the models with the experimental feedback responses intensities to evaluate our models. We do not evaluate models in terms of kinematics or any other variables. As the predictive simulated feedback intensities for each of the four models provided very different patterns, the important comparison is qualitative. However, we supplemented this qualitative comparison with a quantitative model comparison using the Bayesian information criterion (BIC). BIC is a conventional method for model comparison which evaluates the log-likelihood of the model fitting to the data while controlling for over-fitting by penalizing additional model parameters (Schwarz, 1978). A BIC difference of 10 is very strong evidence for the model with the lower BIC. Overall, we used individual participant mean feedback intensities for baseline, earlypeak hand velocity and late-peak hand velocity conditions, providing us with a total of 150 data points (10 participants $\times$ three conditions $\times$ five perturbations) to determine the fit. Moreover, to compare the captured variance of the data between our OFC models and the timeto-target tuning curve we also calculated the sum of squared-residuals (SSRs) between the models and the data.

\section{Results}

\section{Experimental results}

In this study, we examine the relation between time-totarget (the time difference between the perturbation onset and the cursor intercepting the target) and the visuomotor feedback responses. To do so, we devised an experiment consisting of five different kinematic conditions. The baseline condition required movements with a natural, bell-shaped velocity profile, while the velocity profiles were modified for the four other conditions. In these four conditions, we introduced a manipulation between the hand velocity and the cursor velocity in the forward direction, such that the cursor and hand had different velocity profiles, but their positions matched at the start and end of the movement (Fig. 2). Two of these four conditions (matched-cursor conditions) required different kinematics of the physical movement to successfully complete the task, but the cursor velocity profiles matched the baseline. This manipulation of hand velocity profiles also resulted in different times-to-target at the same distance in the movement. The two other conditions (matched-hand conditions) required the same hand movement as for the baseline condition, but as a result the cursor moved with different velocity profiles (see Materials and Methods). This manipulation of the cursor velocity profiles separates the relative contributions of physical and visual hand information in regulating the feedback responses. For each condition we measured the visuomotor feedback intensities (mean corrective force applied during the 180 to $230 \mathrm{~ms}$ time window after a visual perturbation) at five different locations in the movement (Fig. 3A). Overall, our paradigm allowed us to modulate the times-to-target across conditions, as well as separate proprioceptive (hand) and visual (cursor) kinematics to examine their individual contribution to visuomotor feedback responses.

Different movement conditions exhibited differences in visuomotor feedback intensities (Fig. 3). Two-way repeated-measures ANOVA (both frequentist and Bayesian; Materials and Methods) showed significant main effects for both condition $\left(F_{(4,36)}=10.807, p<0.001\right.$, and $\left.B F_{10}=9.136 \times 10^{12}\right)$, and perturbation location $\left(F_{(4,36)}=\right.$ 33.928, $p<0.001$, and $\left.B F_{10}=6.870 \times 10^{9}\right)$. Post hoc analysis on movement conditions revealed significant differences between baseline (gray line) and matched-cursor late-peak hand velocity condition (blue line; $t_{(9)}=4.262$, $p_{\text {bonf }}<0.001$ and $\left.B F_{10}=247.868\right)$, and between baseline and matched-cursor early-peak hand velocity condition (green line; $t_{(9)}=-8.287, p_{\text {bonf }}<0.001$ and $B F_{10}=1.425 \times$ 108). However, no significant differences were found between the baseline and the two matched hand velocity conditions $\left(t_{(9)}=1.342, p_{\text {bonf }}=1.0\right.$ and $B F_{10}=0.357$ for early-peak cursor velocity, yellow; $t_{(9)}=0.025, p_{\text {bonf }}=1.0$ and $B F_{10}=0.154$ for late-peak cursor velocity, purple). Our results show that different kinematics of the hand movement have a significant effect on visuomotor feedback response regulation, but that different kinematics of the cursor movement do not.

One possible explanation for differences between the two matched-cursor conditions (Fig. $3 \mathrm{C}$, blue and green) and the baseline condition (gray) might arise from a different mapping between cursor and hand velocities (Fig. 2A) that had to be learned. Alternatively, the incongruency between the vision and proprioception might be another explanation. However, the two matched-hand conditions (yellow and purple) had the identical mappings (and incongruencies) as the two matched-cursor conditions (blue and green, respectively) and yet no differences were found in these conditions. Instead, the only conditions in which differences in the feedback gains were found, were conditions in which the timing of the peak hand velocity was shifted.

In order to test whether a simple relationship between movement kinematics and visuomotor feedback intensities exists, we mapped visuomotor feedback intensity magnitudes as a linear function of the hand velocity and the cursor velocity. For each experimental condition, we find a different regression slope between the velocity and the feedback intensities regardless of whether this is the cursor or the hand velocity (Fig. $4 A, B$ ). Consistent with our previous results, this difference in slopes is significant for conditions where the hand, but not cursor, movement was different (Fig. 4C,D). Although feedback intensities increase with increasing velocity in both cursor and hand coordinates, no one coordinate modality could predict the changes in the feedback intensity.

To successfully complete each trial, participants were required to reach the target. However, the distance to reach the target is affected by the perturbation onset, 

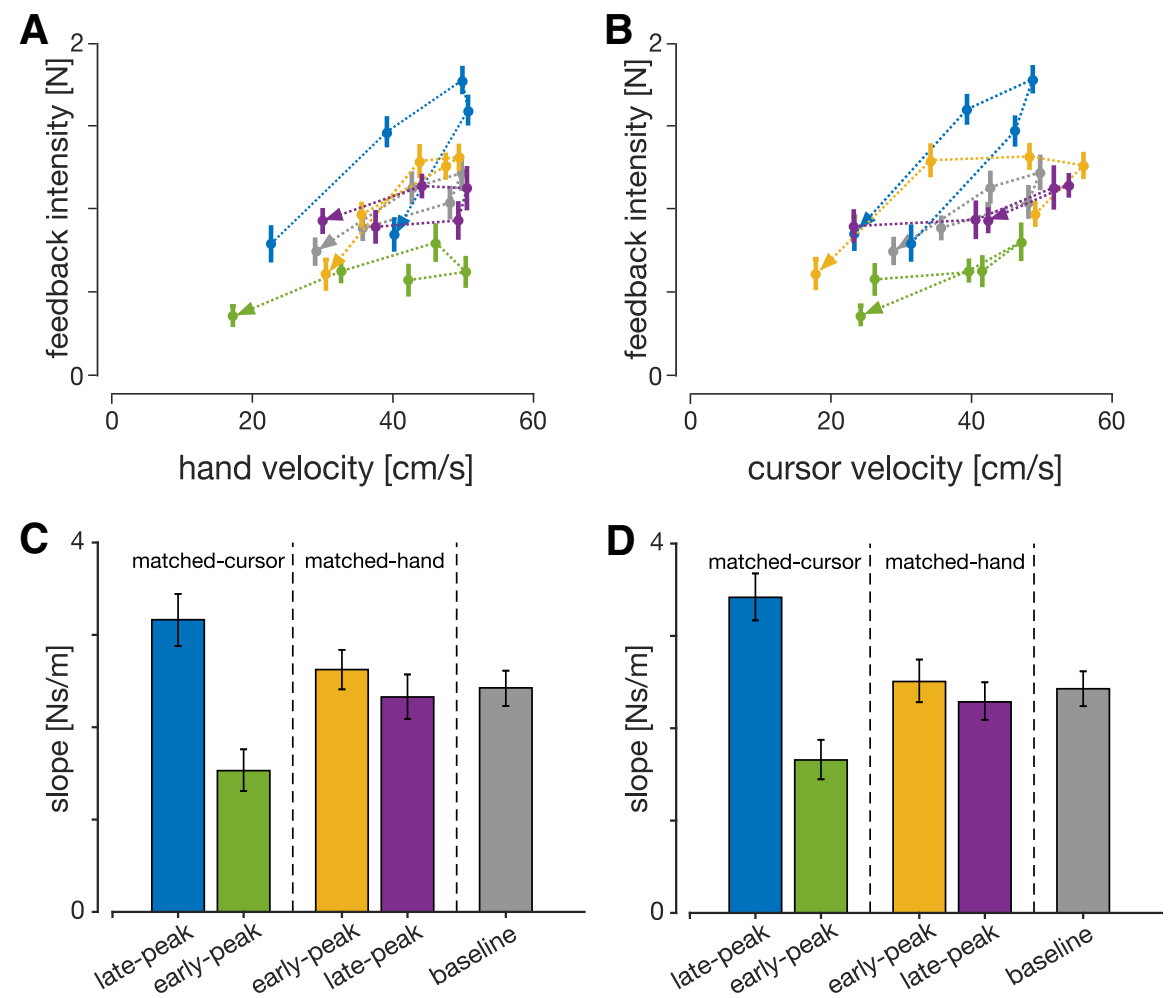

Figure 4. Visuomotor feedback intensities as a function of $(\boldsymbol{A})$ hand velocity and $(\boldsymbol{B})$ cursor velocity at the time of perturbation for all experimental conditions. Error bars represent 1 SEM, and the arrowheads represent the order of the perturbation locations. $\boldsymbol{C}$, $\boldsymbol{D}$, Regression slopes of feedback intensities for each condition as a function of hand and cursor velocities, respectively. Error bars represent $95 \%$ confidence intervals of the slopes. The slopes for the two matched-cursor conditions were significantly different (based on the confidence intervals) than for the baseline condition.

later perturbation locations lead to larger correction angles (Fig. 5A) and thus longer movement distances (Fig. $5 B$ ). That is, an earlier correction means that the trajectory can go directly toward the target, whereas a later correction would require a new corrective movement and therefore further distance. This effect is clearly seen where the extension of movement distance is enhanced for the perturbations closest to the target, with movement distance extended by almost $0.5 \mathrm{~cm}$ compared with less than 1 $\mathrm{mm}$ for the closest perturbations. Any extension of the movement distance requires an appropriate increase in movement duration. Consequently, participants extended their movement time, with longest durations for perturbations close to the target (Fig. 6A). This increase in movement duration increases the time-to-target for these late perturbations (Fig. 6B), and now allows sufficient time for the controller to issue any corrective commands.

\section{Finite horizon OFC}

As optimal control has been suggested to predict the temporal evolution of feedback intensities (Liu and Todorov, 2007; Dimitriou et al., 2013), we built two finitehorizon OFC models: the classical model (Liu and Todorov, 2007), and a time-to-target model. For the classical model we implemented an OFC (Todorov, 2005) to simulate movements with different velocity profiles, similar to the experiments performed by our participants. We extended this classical model to the time-to-target model, by increasing the movement duration after each perturbation onset according to experimental results (Fig. 6). For both models we only simulated different hand kinematics for computational ease and as our participants showed little effect of cursor kinematics on their feedback intensities.

For both models we controlled the activation cost $\mathrm{R}$ to simulate three conditions in which the location of the peak velocity was shifted to match the experimental hand kinematics (Fig. 7A). Specifically, we solved for the activation cost $\mathrm{R}$ and movement duration $\mathrm{N}$ by optimizing the loglikelihood of our model's peak velocity location and magnitude using BADS (Acerbi and Ma, 2017). The optimized movement durations (mean \pm SEM) were $\mathrm{N}=930 \pm 0 \mathrm{~ms}$ for the baseline condition, $N=1050 \pm 10 \mathrm{~ms}$ for the latepeak condition and $\mathrm{N}=1130 \pm 20 \mathrm{~ms}$ for the early-peak condition (10 optimization runs per condition). In comparison, experimental movement durations were $\mathrm{N}=932 \pm$ $30 \mathrm{~ms}$ for the baseline condition, $\mathrm{N}=1048 \pm 47 \mathrm{~ms}$ for the late-peak condition and $1201 \pm 59 \mathrm{~ms}$ for the early-peak condition, matching well with the OFC predictions. Overall, this shows that specific constraints on the magnitude and location of peak velocity that we imposed on our participants resulted in a modulation of reaching times that matched OFC predictions under the same constraints.

For the classical model we estimated simulated feedback intensities by shifting the movement target at each time point in the movement and measuring the mean 
A

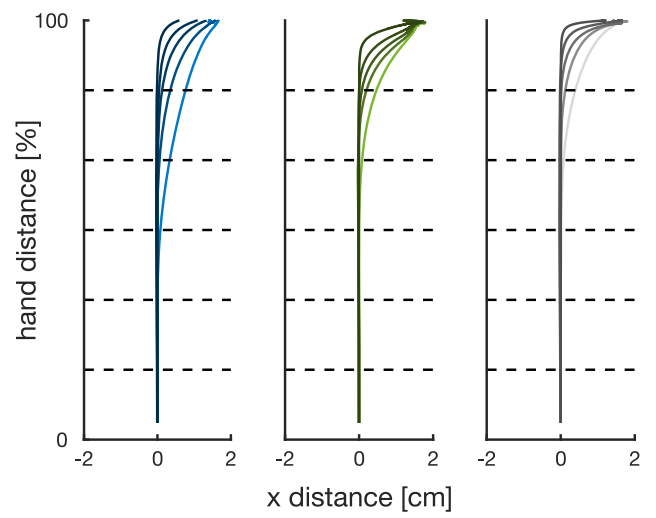

B

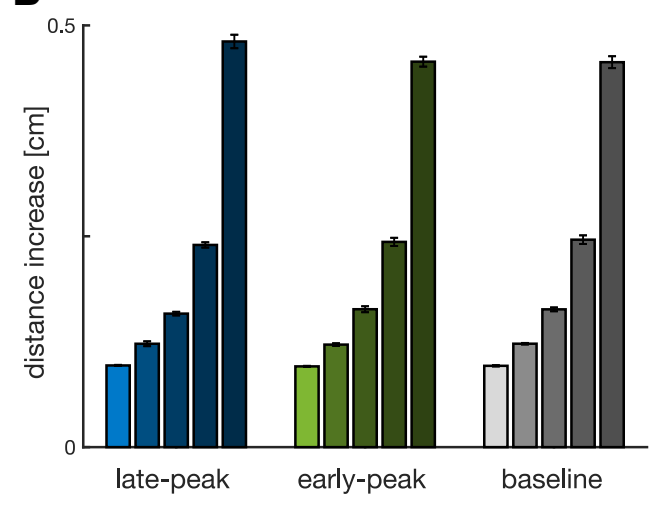

Figure 5. A, Mean hand movement trajectories for matched-cursor late-peak (left), matched-cursor early-peak (middle), and baseline (right) conditions recorded in our participants, with perturbation onset at five locations [color light to dark: $4.2 \mathrm{~cm}(16.7 \%)$, $8.3 \mathrm{~cm}(33.3 \%), 12.5 \mathrm{~cm}(50 \%), 16.7 \mathrm{~cm}(66.7 \%)$, and $20.8 \mathrm{~cm}(83.4 \%)$ from the start position; dashed lines]. Corrections to rightward perturbations were flipped and combined with leftward corrections. $\boldsymbol{B}$, Distance increase for each perturbation location recorded in our participants. Perturbation locations closest to the target required the largest increases in movement distance. Error bars represent 1 SEM.

magnitude of the simulated force response over a 130- to 180 -ms time window in the direction of this shift. The simulated feedback intensity profiles follow the same general shape as in human participants, intensity increases from the beginning of the movement and then falls off at the end (Fig. 7B). However, the overall profile of these simulated feedback intensities is very different for each of the kinematic conditions. For the early-peak velocity condition, the simulated feedback intensity peaks toward the end of the movement (green line), whereas for the latepeak velocity condition the simulated feedback intensity profile peaks early in the movement (blue line). These simulated feedback intensities do not appropriately capture the modulation of visuomotor feedback intensities in our experimental results. Specifically, they predict a temporal shift in the peak intensity that is not present in our participants data, and predict similar peak levels of feedback intensities across all three conditions. While the simulated feedback intensities are qualitatively similar to the experimental results within each condition; overall, this model cannot appropriately capture the modulation of visuomotor feedback responses across the conditions.

For the time-to-target OFC model, we extended the classical model to account for the different movement durations for each perturbation location (and movement condition) that is seen in the experimental results. After a
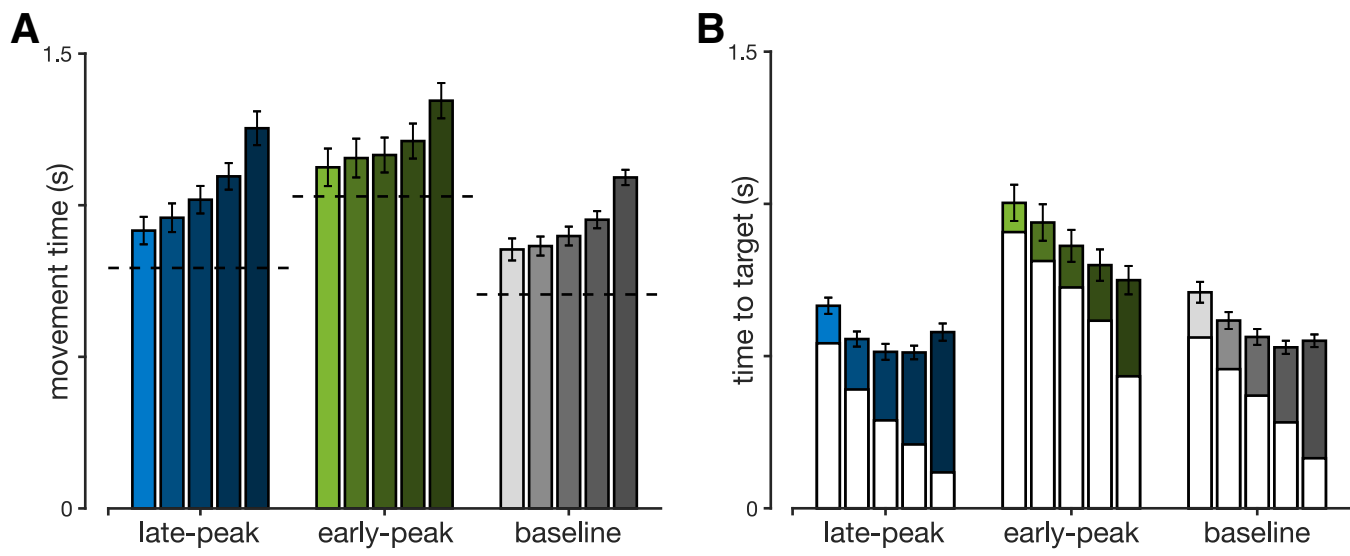

Figure 6. $\boldsymbol{A}$, Movement durations in maintained perturbation trials recorded by our participants in late-peak, early-peak and baseline conditions. Separate bars within the same color block represent different perturbation onset locations (left to right: 4.2, 8.3, 12.5, 16.7 , and $20.8 \mathrm{~cm}$ from the start position). Error bars represent $1 \mathrm{SEM}$ while the horizontal dashed lines represent movement durations in the same movement condition for non-perturbed movements. B, Full bars represent times-to-target (time between a perturbation onset and target interception) in maintained perturbation trials in our participants for late-peak, early-peak, and baseline conditions. White bars represent the time-to-target for a respective non-perturbed movement, at the time when the perturbation would have happened. The colored part of the bars represents the extension in times-to-target due to the perturbation in a non-constrained movement. This shows that the perturbation during the movement evokes an extension in the time-to-target and subsequently in movement duration Each of the five bars represents a different perturbation onset location, as in $\boldsymbol{A}$. Error bars represent 1 SEM. 

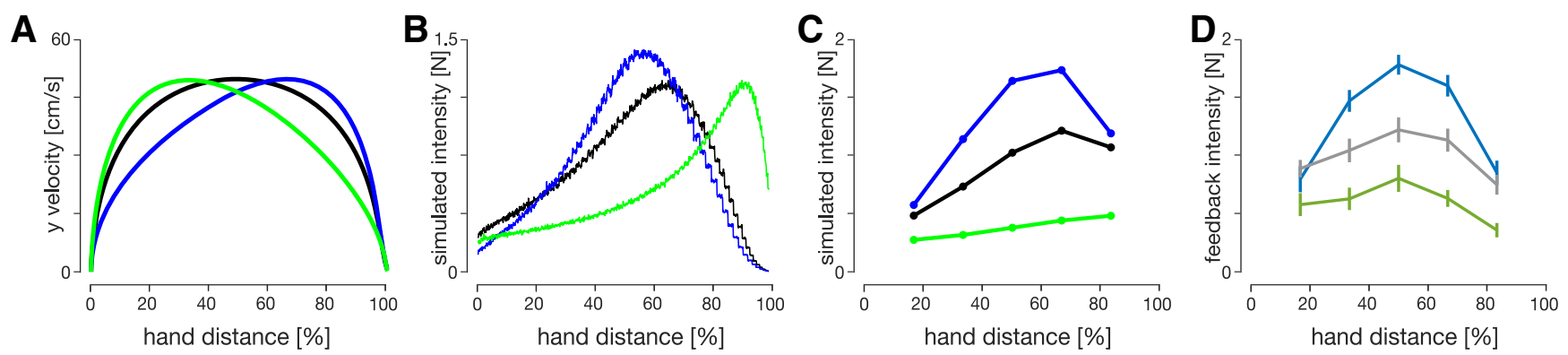

Figure 7. Comparison of feedback intensities between the two OFC models and experimental data. Simulated velocity profiles $(\boldsymbol{A})$ and simulated feedback intensity profiles $(\boldsymbol{B})$ of baseline (black), early-peak (green), and late-peak (blue) velocity condition simulations for the classical OFC model. Velocity profiles were obtained by constraining the velocity peak location and magnitude and optimizing for movement duration and activation cost function. Simulated feedback intensity profiles were obtained by applying virtual target jumps perpendicular to the movement direction during these movements and calculating the force exerted by the controller in the direction of the target jumps. The jagged appearance of the intensity traces is simply an outcome due to the simulation time step. $\boldsymbol{C}$, Simulated feedback intensities obtained via the time-to-target OFC model. Preperturbation movements were simulated as if no perturbation would occur, to keep the controller naive to an upcoming perturbation. At the perturbation onset the remaining movement duration is adjusted to match the mean time-to-target for a similar perturbation onset in human participants (Fig. 6B). Therefore, this model only simulates the feedback intensities at the five perturbation locations in the movement. The velocity profiles for the time-to-target model match the velocity profiles of the classical model, shown in $\boldsymbol{A}$. $\boldsymbol{D}$, Visuomotor feedback intensities recorded in human participants.

perturbation, the remaining time-to-target was adjusted to match the experimentally recorded times-to-target for this specific movement, while before the perturbation both the classical model and the time-to-target model were identical. After adjusting for the individual durations of each perturbation condition we are now able to qualitatively replicate the general regulation of feedback intensity profiles for different kinematics using OFC (Fig. 7C). In the late-velocity peak condition we predict a general increase in the feedback responses throughout the movement compared with the baseline condition, whereas in the early velocity peak condition we predict a general decrease in these feedback responses compared with the baseline condition. Thus, we show that within the OFC the time-to-target is critical for the regulation of feedback responses, and when we take this into account, we are able to replicate the feedback intensity modulation of our participants.

While in our experiment we manipulated the time-totarget through skewing the velocity profiles, time-to-target is naturally modified through changing the peak velocity. Therefore, we can further analyze the effect of the time-to-target by calculating the feedback intensities for movements with different peak velocities (Fig. 8A). The simulated feedback intensities vary widely across peak velocities, with a shift of peak feedback intensities toward the earlier locations for faster movements (Fig. 8B). However, when these distinct simulated feedback intensity profiles are re-mapped as a function of time-to-target, the simulated feedback intensities follow a consistent, albeit non-monotonic, relationship (Fig. 8C). This relationship is also consistent over a range of peak velocities across all three kinematic conditions and is well described by a combination of a square-hyperbolic and logistic function (Fig. 8D). The squared-hyperbolic arises from the physics of the system: the lateral force necessary to bring a point mass to a target is proportional to $1 / t^{2}$ (Materials and Methods; Eq. 9). The logistic function simply provides a good fit to the data. Overall, our models show that the feedback intensity profiles under OFC are independent of the peak velocity or movement duration. Instead, our simulations suggest that time-to-target is a key variable in regulating visuomotor feedback responses.

It has been shown that the optimal controller gains (Liu and Todorov, 2007), as well as the visuomotor feedback intensities (Knill et al., 2011; de Brouwer et al., 2017) are influenced by task definition (e.g., instruction to hit the target or stop at the target). Here, we simulated the hit, fast hit and stop instructions for our classical model to test how it influenced the relation between simulated feedback intensity and time-to-target. Our previous simulations represent the stop instruction. We modified the $\omega_{v}$ and $\omega_{f}$ to simulate the baseline equivalent of hit and fast hit instructions. Specifically, we set $\omega_{v, \text { hit }}=\omega_{v} / 4=0.05$, $\omega_{f, \text { hit }}=\omega_{f} / 4=0.005$ for hit instruction, and $\omega_{v, \text { fasthit }}=$ $\omega_{v} / 10=0.02, \omega_{f, \text { fasthit }}=\omega_{f} / 10=0.002$ for fast hit instruction. As changing the terminal costs also results in a change in peak velocity, we further reduced the desired movement times to $\mathrm{N}=800 \mathrm{~ms}$ for the hit instruction and $\mathrm{N}=750 \mathrm{~ms}$ for fast hit instruction, such that all three peak velocities match (Fig. 9A). According to our simulations, such modification of task demands produced different simulated feedback intensity profiles (Fig. 9B). However, the intensity relationship with time-to-target maintained the same structural profile independent of the task demand (Fig. 9C). Specifically, both the squared-hyperbolic and logistic segments of the control are still present, although we observe the shift in the temporal location of the crossover point. While each task requires a different pattern of feedback gains (and will therefore produce different responses), variations of the kinematic requirements within a task do not change these gains and therefore do not require recalculation.

\section{Receding horizon and infinite horizon control}

A limitation of the finite-horizon implementation used in classical and time-to-target models is that the variable movement duration (Fig. 6) is the model input rather than 

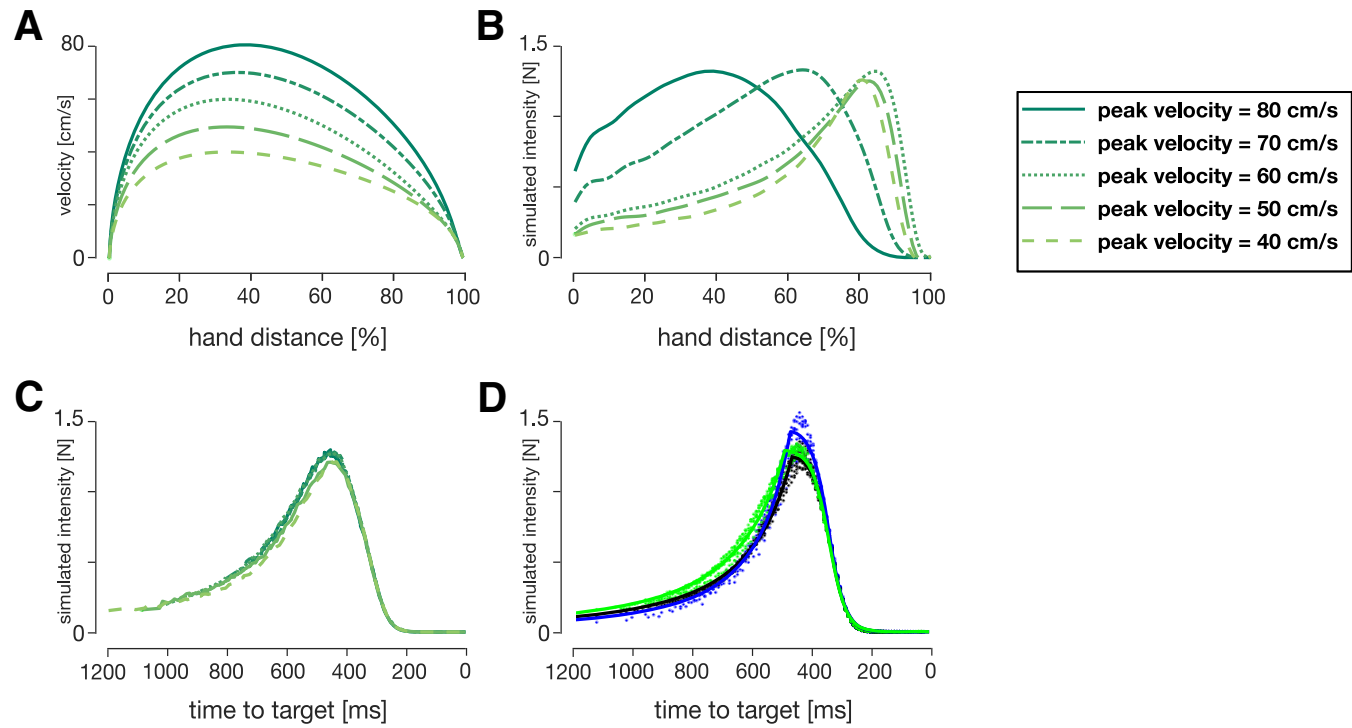

Figure 8. OFC simulations of $(\boldsymbol{A})$ velocity profiles and $(\boldsymbol{B})$ simulated feedback intensity profiles for different desired peak velocities (in order from light to dark line colors: $40,50,60,70$, and $80 \mathrm{~cm} / \mathrm{s}$ ). C. Simulated feedback intensities of (B) re-mapped as a function of time-to-target at the time of perturbation. $\boldsymbol{D}$, Simulated feedback intensities vs time-to-target for the three kinematic conditions over the five peak velocities simulated by OFC (colored dots). Solid lines represent the tuning curves (Eq. 7) fit to the data. Both the tuning curves and the simulated feedback intensity profiles are similar across a variety of different kinematics when expressed as a function of time-to-target.

output. Therefore, in addition to finite-horizon models we also modeled our task in receding and infinite horizon for a single movement condition. Specifically, for the infinite horizon model both state-dependent and regulator costs were kept constant throughout the simulated movement. For the receding horizon model, the regulator cost was kept constant, while the state-dependent cost was zero for all but last "foreseeable" state. Such models were expected to simulate the baseline experimental condition, however the resultant velocity profile better resembled the early-peak condition (Fig. 10A). As a result, we compared these simulations with both baseline and earlypeak velocity condition data and with the time-to-target model simulations (Fig. 10B-D).

Both receding horizon and infinite horizon LQG models were able to successfully capture the nonlinear change in trial durations for different perturbation onsets (Fig. 10B) matching the experimental results. In addition, these models also predicted variable times-to-target for the five perturbation onset locations: $700,660,620,600$, and
$580 \mathrm{~ms}$ for the infinite horizon and $690,640,610,610$, and $600 \mathrm{~ms}$ for the receding horizon. However, neither model showed variation of the simulated feedback intensities for different perturbation onset locations (Fig. 10C,D), a result that was present in the experimental data and captured by our time-to-target model. Instead both models predicted constant feedback intensities for all perturbations locations. Therefore, neither the receding nor the infinite horizon models are able to explain our experimental results. While both of the approaches can accurately capture the variability in movement duration, only the time-totarget model well describes the behavioral variation in visuomotor feedback responses.

\section{Quantitative model comparison}

Qualitatively, our results suggest that the time-to-target is an important variable when correcting for visual perturbations in a visuomotor task. In order to supplement these findings quantitatively, we also evaluated model fits
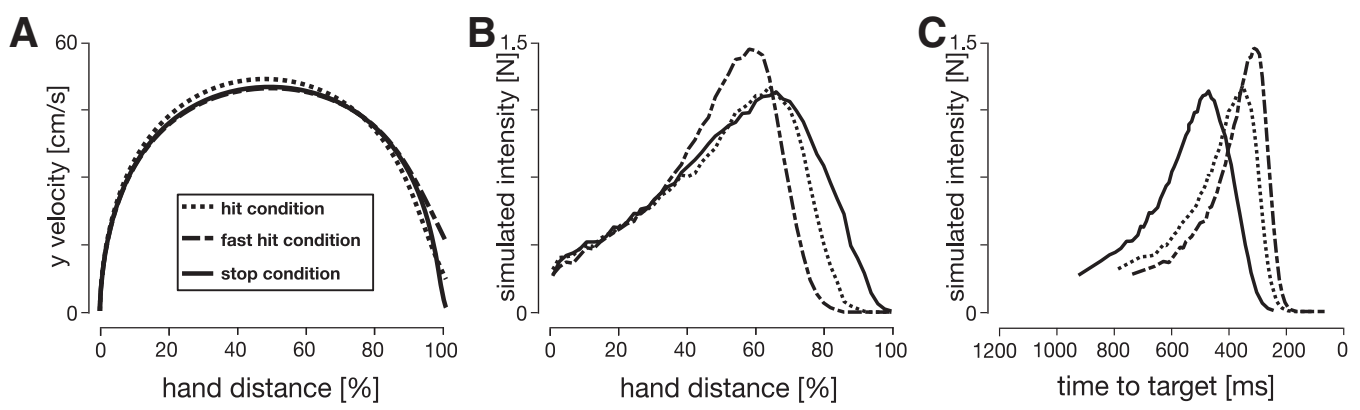

Figure 9. Comparisons between hit and stop instructions. $\boldsymbol{A}$, Velocity profiles for the stop, hit and fast-hit conditions. $\boldsymbol{B}$, Simulated feedback intensity profiles as a function of hand position. $\boldsymbol{C}$, Simulated feedback intensities of $(\boldsymbol{B})$ re-mapped as a function of timeto-target at the time of target perturbation. 
A

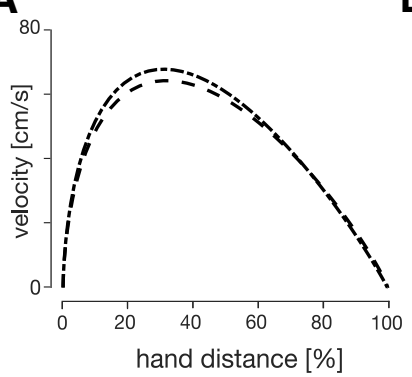

B

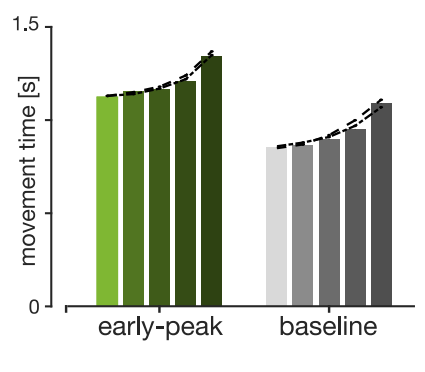

C

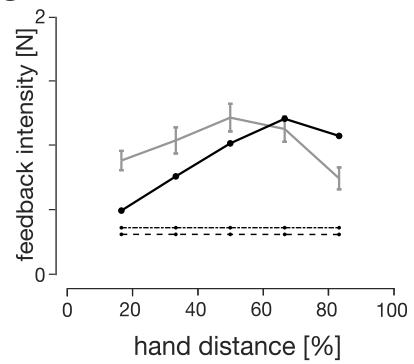

D

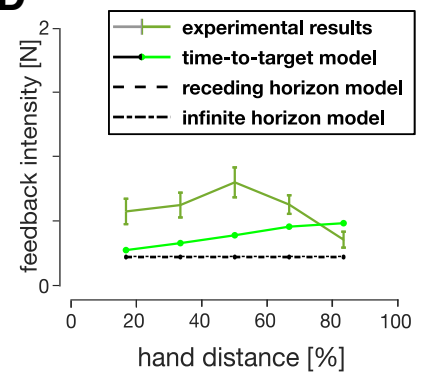

Figure 10. Receding horizon and infinite horizon model simulations. $\boldsymbol{A}$, Simulated velocity profiles of receding horizon (dashed) and infinite horizon (dot-dashed) models. Both models naturally produce positively skewed velocity profiles, more closely resembling early-peak velocity, rather than the baseline condition. $\boldsymbol{B}$, Mean experimental movement durations (bar chart) compared with the receding and infinite horizon model predictions. Both models accurately simulate the variations in the reach durations with perturbation location. Baseline $(\boldsymbol{C})$ and early-peak velocity condition $(\boldsymbol{D})$ simulations for receding horizon, infinite horizon and time-to-target (dot-solid lines) models, compared with the experimental data. Only the time-to-target model predicts different visuomotor feedback response intensities for different perturbation onset locations, while receding and infinite horizon models predict constant intensities. Note that models were not fit to match the intensities, only to qualitatively demonstrate the behavior.

between the data and the models using BIC and calculating the SSR for each of the models. We compared the OFC-based models with respect to the classical, finite horizon OFC model as our baseline model. Consistent with our qualitative estimations, the time-to-target OFC model performed the best of all OFC-based models $\left(\Delta B / C_{t t t}=\right.$ 38.2). In addition, both receding-horizon and infinite-horizon models provided bad fits to the data $\left(\triangle B / C_{\text {rec }}=\right.$ $\left.-23.4, \Delta B / C_{\text {inf }}=-18.4\right)$. We also compared, using SSR, the fit of our OFC models with the simpler time-to-target tuning curve (Eq. 7; Fig. 11A). While the tuning curve is only a simple approximation to the time-to-target adjusted OFC feedback predictions, it is still able to explain a similar amount of variance in the data $\left(R^{2}=0.33 ; S S R_{\text {tuningcurve }}=28.5\right.$, $S S R_{\text {classical }}=33.3, S S R_{\text {ttt }}=24.9, S S R_{\text {inf }}=S S R_{\text {rec }}=38.9$ ). Overall, both the BIC and SSR comparison confirms that the time-to-target OFC model best explains the feedback modulation during human reaching and suggests that time-to-target is a critical variable in online control.

Overall, our simulations suggest that, independent of movement kinematics (different temporal position, velocity, and acceleration profiles), the visuomotor feedback intensities follow the same profile with respect to the time-to-target. We further verified how our time-to-target prediction matches our actual experimental results by plotting participants' visuomotor feedback intensities against the average time-to-target for the respective perturbation locations and movement conditions (Fig. 11A). Specifically, the intensities monotonically increase with decreasing time-to-target until the peak (following the squared-hyperbolic function) and then reduce (the logistic function range).

\section{Validation of the time-to-target model}

We also compared the prediction of the time-to-target model to independent results from an external data set (Dimitriou et al., 2013). In the article, the authors could not rigorously encapsulate both conditions within a simple relationship to movement distance, movement fraction or movement velocity. We plotted visuomotor feedback intensities against time-to-target for two experimental conditions: goal directed reach of $17.5 \mathrm{~cm}$ and of $25 \mathrm{~cm}$ (Fig. 11B,C). Two observations can be made from these results. First, the time-to-target model prediction and the experimental data follow the same qualitative features, independent of the target distance (experimental condition). Second, the feedback intensities for both conditions are well explained by a single relationship with time-to-target (Fig. $11 C ; R^{2}=0.56, S S R_{\text {tuning curve }}=2.3$ ). Thus, the validation against an external dataset supports our results that visuomotor feedback intensities vary with the timeto-target.

Finally, we evaluated our optimal control models (the classical and the time-to-target) on this dataset. Similar to our original fitting, we fit both of our OFC models to match the kinematics of the human participants, and then simulated the virtual experiment to extract simulated feedback intensities for the models. We found that model parameters $\overrightarrow{\omega_{p}}=[0.5,1], \omega_{v}=0.03$, and $\omega_{f}=0.03$ and $R(t)=0.00000235$ provide the best fit of kinematics between OFC models and data. As with our data, the time-to-target OFC model provided a better fit of the simulated feedback gains than the classical OFC model $\left(\Delta B I C=19.2, S S R_{t t t}=8.6, S S R_{\text {classical }}=10.7\right)$. Together, both our data and Dimitriou et al. (2013) data strongly support our time-to-target model.

\section{Discussion}

Here, we examined how movement kinematics regulate visuomotor feedback responses. Participants extended their movement duration after perturbations to successfully reach the target. In addition, visuomotor feedback responses were modulated when the hand followed different kinematics, but not when the cursor followed different kinematics. In order to better understand this modulation we built four normative models using OFC: a classical finite-horizon OFC (Liu and Todorov, 2007), a finite-horizon time-totarget adjusted OFC, a receding-horizon OFC (Guigon et al., 2019), and an infinite-horizon OFC (Qian et al., 2013). While the classical, receding and infinite horizon models failed to predict the experimental visuomotor feedback 

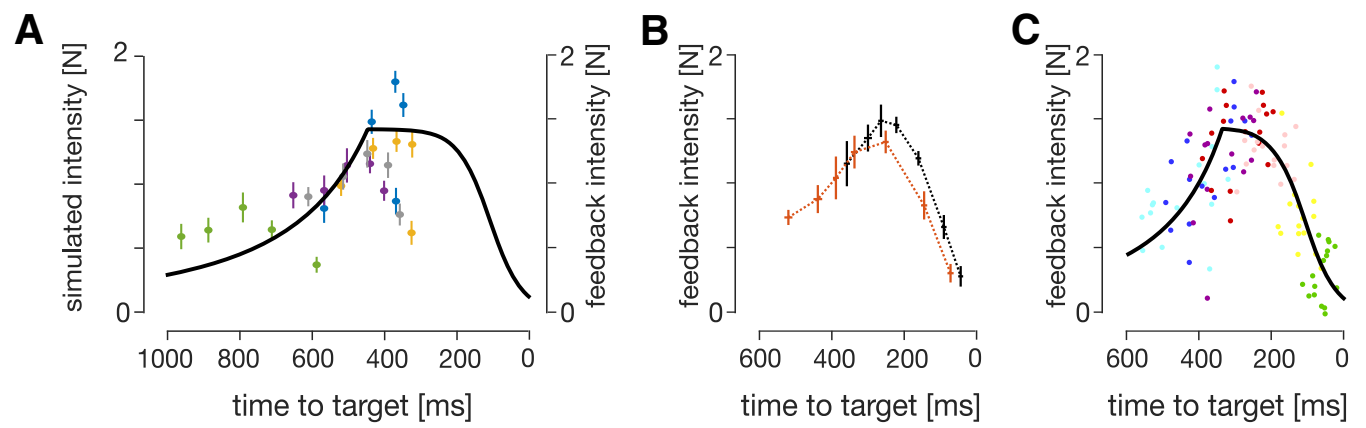

Figure 11. Validation of the time-to-target model. A, Experimental visuomotor feedback intensities for all five experimental conditions (scatter plot) overlaid with the time-to-target tuning curve. The data and the tuning curve show similar qualitative features. Error bars represent 1 SEM. Marker colors indicate five experimental conditions as described in Figure 2B. B, Experimental data of the visuomotor feedback intensities of Dimitriou et al. (2013), mapped against the time-to-target. Black and orange traces represent mean participant data for 17.5 and $25 \mathrm{~cm}$ movement conditions, respectively. $\boldsymbol{C}$, A scatter plot of individual subjects' data from $\boldsymbol{B}$, overlaid by the time-to-target tuning curve. Both, 17.5 and $25 \mathrm{~cm}$ movement conditions are combined to a single representation. Different colors represent different perturbation onset distances as in Dimitriou et al. (2013).

response intensities, the time-to-target model qualitatively replicated the visuomotor feedback intensity profile of our participants. Overall, OFC models suggested that feedback intensities for each perturbation location depended on the time-to-target (i.e., time between perturbation onset and target interception) rather than distance or velocity. Moreover, this explains why any mismatch between visual and haptic kinematics had no effect on the feedback intensities, as these manipulations did not affect the time-to-target. Simulated feedback intensities under all movements followed the same profile with respect to time-to-target, suggesting a critical role in the regulation of visuomotor feedback responses.

Experimentally, our participants exhibited a temporal evolution of visuomotor feedback intensities for each condition, confirming the findings of Dimitriou et al. (2013). In addition, we also showed the regulation of visuomotor feedback responses across conditions, allowing us to investigate the underlying mechanism of this temporal evolution. Specifically, our experimental results demonstrated strong regulation of visuomotor feedback intensity profiles with different hand kinematics, but not with different cursor kinematics (Fig. 3C). Compared with the baseline condition, in the matched-cursor early-peak velocity condition participants produced longer times-to-target at each perturbation location (Fig. 6B), resulting in weaker feedback responses based on the relationship between time-to-target and visuomotor feedback intensities (Fig. 11A). The opposite is true for the matched-cursor late-peak velocity condition. As the two matched-hand conditions produced similar times-to-target as the baseline due to similar hand kinematics, we did not observe a different regulation in feedback responses. Therefore, the condition-dependent visuomotor feedback response modulation exhibited by our participants meshes nicely with a control policy whereby the time-to-target regulates the feedback responses.

It has long been suggested that we select movements that minimize the noise or endpoint variability (Harris and Wolpert, 1998). Within the framework of optimal control, this idea has been expanded to the corrective movements, that is, optimality in reaching movements is achieved in part by minimizing the noise during any corrective response (Todorov and Jordan, 2002). As motor noise scales proportionally to muscle activation (Jones et al., 2002; Hamilton et al., 2004), one way of minimizing such noise is reducing the peak levels of muscle activation during the correction. Mathematically, the optimal solution to correct any perturbation approximates a constant activation, resulting in a constant force for the whole duration between perturbation onset and target interception. Such a solution assumes that the brain is capable of estimating the remaining duration of the movement (Mclntyre et al., 2001; Benguigui et al., 2003; Zago et al., 2004) and that the force follows the squared-hyperbolic relationship to this duration (Eq. 9). The parallel can be drawn here between our results and the results of Oostwoud Wijdenes et al. (2011), where the authors showed a similar temporal evolution of peak acceleration against the time-to-target in a single forward velocity condition. Our results further show that time-totarget strongly modulates visuomotor feedback responses across a range of different kinematics, consistent with the idea that human participants aim to behave optimally. More specifically, we suggest that, among different optimality variables, the temporal evolution of visuomotor feedback response intensities serves to reduce effects of system noise.

Finite-horizon OFC predicts a time beyond which feedback responses are suppressed. Beyond this critical time, a logistic function well describes the relation between time-to-target and feedback responses, with response intensities reducing as the time-to-target decreases. The controller gains at this stage are the most sensitive to acceleration, suggesting a more "behavioral" outcome, the controller is trying to stop, rather than correct errors. The neural recordings in rhesus macaque monkeys' supplementary motor area and M1 (Russo et al., 2019) show that supplementary motor area can signal movement termination as far as $500 \mathrm{~ms}$ before the end of the movement. This further suggests that there may be multiple stages within a movement, where our control system might "care" more about error correction in one or movement termination in another. On the other hand, the suppression of responses 
close to the target leads to undershooting the target. Our participants, however, had to bring the cursor to the target to advance to the next trial. As a result, they extended the movement durations postperturbation to return to the squared-hyperbolic range of control. The control performance of such behavior is well accounted for by our timeto-target model. Moreover, our time-to-target model also well explained the modulation of visuomotor feedback intensities from an external data set (Dimitriou et al., 2013). However, an important distinction from our study is that in Dimitriou et al. (2013), the suppression of feedback responses toward the end of movements would not interfere with reaching the target as perturbation trials were always in a mechanical channel so that no corrections were required. As a result, the times-to-target were shorter and the data clearly exhibits both logistic and squaredhyperbolic segments of the control.

All of the variations of optimal control models are formulated as two controllers in $x$ - and $y$-axes (coupled through control-dependent noise), with no modeling of the musculoskeletal dynamics. However, the experiments were performed using multijoint reaching movements of the arm. One possibility is that the presence of these musculoskeletal dynamics in the human participants could explain the differences in the feedback intensities, as the matched cursor conditions required different hand accelerations. One might therefore imagine that the condition with the fastest initial movements (early-peak velocity) requires the largest initial forces and could therefore produce larger initial feedback intensities as a default. However, several studies have shown that there is no scaling of visuomotor feedback gains with background loads or muscle activity (Franklin et al., 2012, 2017). More critically, this condition actually shows the lowest feedback gains early in the movement, whereas the condition with the slowest initial acceleration produces the highest feedback gains. Therefore, we suggest that the neuromuscular dynamics cannot explain the modulation of these feedback responses.

Both of the matched cursor conditions (early and late peak) require a change in the physical kinematics away from the naturally occurring bell-shaped velocity profile. One possibility is that this manipulation could have driven the changes in feedback intensity. We argue against this possibility for two reasons. First, all participants were able to fairly quickly learn this pattern of movement with training before the testing of the feedback intensities. Second, if this manipulation away from the naturally occurring bellshaped profile affects these feedback intensities, we would expect the feedback gains in these two conditions to either both increase or both decrease. Instead we find that the feedback intensity profile for each condition changes in a manner that is explained by the change in the time-to-target.

A limitation of our time-to-target model is that it takes time-to-target as an input to generate feedback intensity predictions, rather than obtain the time-to-target as a model output. As a result, our time-to-target model does not describe exactly how the change in movement geometry after the perturbation influences this time-to-target, which in turn regulates the visuomotor feedback responses. On the other hand, both receding and infinite horizon models did predict the movement duration change after perturbations very well, but could not at all describe the changes in visuomotor response intensity. However, utility of movement has recently been used within optimal control to characterize reaching movements (Rigoux and Guigon, 2012; Shadmehr et al., 2016) in which optimal movement time falls out automatically from a trade-off between reward and effort. With respect to our models, this adds additional complexities to capturing the different movement conditions. Future approaches could attempt to model these results within the utility of movement framework.

In addition, our time-to-target model does not directly show the causality of the time-to-target as a control variable for the visuomotor feedback intensities. Particularly, the time-to-target relation to feedback intensity could be a byproduct of a more sophisticated control scheme. Additional arguments for the time-to-target control scheme could be two-fold. First, there is evidence that humans are well capable of estimating the time-to-target of a moving stimulus, even if it is accelerating (Mclntyre et al., 2001; Benguigui et al., 2003; Zago et al., 2004), indicating that time-to-target is at least an available input for such a controller. Second, while we have tested finite-horizon OFC and two other (receding and infinite horizon) OFCs, only the finite horizon controllers had any effect on the variation of simulated feedback intensities. Importantly, neither the receding nor infinite horizon models use time-to-target as an input to the controller. We posit that this time-to-target control input is the one key difference between the finite and non-finite models and is therefore the simplest explanation for our results.

Our results show that models incorporating time-to-target (the time-to-target OFC and the simple time-to-target tuning curve) better describe our experimental data and those of Dimitriou et al. (2013) than do other optimal control models. Specifically, the relative scaling of the conditions is explained using the time-to-target tuning curve (Fig. $11 \mathrm{~A})$ : the times-to-target are longer for the early-peak velocity condition compared with the baseline, and therefore fall in the lower intensity range (and vice versa for the latepeak condition). However, there are still some qualitative differences between the experimental and model predictions. That is, our experimental results exhibited an inverted U-shape for the feedback intensity profiles, whereas the model predicts only a slow increase in some conditions (Fig. $8 \mathrm{C}$, green curve). Our time-to-target model suggests that this inverted $U$-shape is not characteristic of the feedback intensity profile, but is simply an outcome of the experimental design (particularly the reaching duration). Indeed, the time-to-target model makes specific predictions about the feedback intensities for much faster movements, which should not show an inverted U-shape but instead decrease throughout the movement. Our model, therefore, makes strong predictions that can be tested in future studies.

Rapid feedback responses scale with the temporal urgency to correct for mechanical perturbations (Crevecoeur et al., 2013). Here, we have shown that visuomotor feedback 
responses also follow a similar regulation, suggesting that these two systems share the same underlying control policy. Our work further extends this finding of Crevecoeur et al. (2013) by not just showing that temporal urgency affects feedback responses, but explaining the manner in which these responses are regulated with respect to urgency. That is, here, we have shown that for visual perturbations the feedback intensities scale with a squared-hyperbolic of the time-to-target, which is a direct measure of urgency. Moreover, the feedback intensities were rapidly adjusted due to the change in urgency as the task changed. Specifically, when the cursor jumps close to the target, the expected time-to-target is prolonged, and therefore the optimal visuomotor feedback response needs to be adjusted appropriately to this increase in time. Our results show that participants produce a visuomotor response consistent with the actual, postperturbation, time-to-target, as opposed to the expected time-to-target before the perturbation. Therefore, our results not only suggest that similar computations might occur for both stretch and visuomotor feedback response regulation, but also that this regulation originates from task-related OFC.

Our proposed time-to-target model is not meant to contradict the conventional OFC models, but rather show that the OFC could be approximated by a simple time-to-target control. Our work has shown that simulated feedback intensities from OFC exhibit the same underlying pattern as a function of time-to-target over a wide range of movement kinematics, matching well the feedback intensities of our human participants (Fig. 7). As expected, changes in the task goals (e.g., hit vs stop) changed the relation between feedback responses and time-to-target. However, the qualitative features, the squared-hyperbolic and logistic function, remained consistent across these tasks. These results suggest that, for a specific task and known dynamics, we do not need to recalculate the feedback gains before each movement, but instead can access the appropriate pattern as a function of the estimated time-to-target in each movement. Therefore, gain computation in reaching movements may not be a computationally expensive process, but instead could be part of an evolutionary control strategy that allows for rapid estimation of the appropriate feedback gains. Moreover, the fact that both stretch reflex and visuomotor feedback systems exhibit similar control policies despite different sensory inputs, perhaps only sharing the final output pathway, suggests that this simple feedback pathway may be an evolutionary old system. Indeed, several studies have suggested that visuomotor feedback is controlled via a pathway through the colliculus (Corneil et al., 2004; Reynolds and Day, 2012; Gu et al., 2019). Furthermore, it has been suggested that visuomotor feedback responses involve two different phases that are behaviorally different Cross et al. (2019). This might reflect two different pathways, the early through the colliculus and the later through cortex. The nature of our analysis only focuses on the earlier of the two phases, which shows limited sensitivity to environment, but is still sensitive to goal redundancy. We suggest that this limited sensitivity could be the outcome of the time-to-target model in action, providing simplified, yet still flexible control in the early phase of the visuomotor response. Such a system would then only need to be adapted as the dynamics or overall task goals change, allowing for fine tuning of the feedback gains according to changes in the environment (Franklin et al., 2017).

Our results have shown the connection between the visuomotor feedback response regulation and the time left to complete the movement. Specifically, in our human participants we recorded the increase in the time-to-target after the perturbation onset, which consequently increased the movement durations (Fig. 6). This increase was also longer for later perturbations, consistent with previous studies (Liu and Todorov, 2007). According to our normative time-to-target OFC model, the time-to-target alone is enough to successfully regulate visuomotor feedback responses as observed in humans. This result was independent of the physical kinematics of the movement or the onset times of the perturbations. This suggests that there is no recalculation of a control scheme for the rest of the movement after the perturbation, but rather a shift to a different state within the same control scheme. Such findings are consistent with the idea that visuomotor feedback gains are precomputed before the movement, allowing for faster than voluntary reaction times (Franklin, 2016). Moreover, through our results, we gain a deeper insight into how OFC governs these feedback gains, through a straightforward relationship to the estimated time-to-target, based on physics.

\section{References}

Acerbi L, Ma WJ (2017) Practical Bayesian optimization for model fitting with Bayesian adaptive direct search. Adv Neural Inf Process Syst 30:1836-1846.

Benguigui N, Ripoll H, Broderick MP (2003) Time-to-contact estimation of accelerated stimuli is based on first-order information. $J$ Exp Psychol Hum Percept Perform 29:1083-1101.

Corneil BD, Olivier E, Munoz DP (2004) Visual responses on neck muscles reveal selective gating that prevents express saccades. Neuron 42:831-841.

Crevecoeur F, Kurtzer I, Bourke T, Scott SH (2013) Feedback responses rapidly scale with the urgency to correct for external perturbations. J Neurophysiol 110:1323-1332.

Cross KP, Cluff T, Takei T, Scott SH (2019) Visual feedback processing of the limb involves two distinct phases. J Neurosci 39:67516765.

Day BL, Lyon IN (2000) Voluntary modification of automatic arm movements evoked by motion of a visual target. Exp Brain Res 130:159-168

de Brouwer AJ, Jarvis T, Gallivan JP, Flanagan JR (2017) Parallel specification of visuomotor feedback gains during bimanual reaching to independent goals. eNeuro 4:ENEURO.0026-17.2017.

de Brouwer AJ, Gallivan JP, Flanagan JR (2018) Visuomotor feedback gains are modulated by gaze position. J Neurophysiol 120:2522-2531.

Dessing JC, Bullock D, Peper CE, Beek PJ (2002) Prospective control of manual interceptive actions: comparative simulations of extant and new model constructs. Neural Netw 15:163-179.

Dimitriou M, Wolpert DM, Franklin DW (2013) The temporal evolution of feedback gains rapidly update to task demands. J Neurosci 33:10898-10909.

Franklin DW (2016) Rapid feedback responses arise from precomputed gains. Motor Control 20:171-176.

Franklin DW, Wolpert DM (2008) Specificity of reflex adaptation for task-relevant variability. J Neurosci 28:14165-14175. 
Franklin DW, Franklin S, Wolpert DM (2014) Fractionation of the visuomotor feedback response to directions of movement and perturbation. J Neurophysiol 112:2218-2233.

Franklin DW, Reichenbach A, Franklin S, Diedrichsen J (2016) Temporal evolution of spatial computations for visuomotor control. J Neurosci 36:2329-2341.

Franklin S, Wolpert DM, Franklin DW (2012) Visuomotor feedback gains upregulate during the learning of novel dynamics. J Neurophysiol 108:467-478.

Franklin S, Wolpert DM, Franklin DW (2017) Rapid visuomotor feedback gains are tuned to the task dynamics. J Neurophysiol 118:2711-2726.

Gu C, Pruszynski JA, Gribble PL, Corneil BD (2019) A rapid visuomotor response on the human upper limb is selectively influenced by implicit motor learning. J Neurophysiol 121:85-95.

Guigon E, Baraduc P, Desmurget M (2007) Computational motor control: redundancy and invariance. J Neurophysiol 97:331-347.

Guigon E, Baraduc P, Desmurget M (2008) Optimality, stochasticity, and variability in motor behavior. J Comput Neurosci 24:57-68.

Guigon E, Chafik O, Jarrassé N, Roby-Brami A (2019) Experimental and theoretical study of velocity fluctuations during slow movements in humans. J Neurophysiol 121:715-727.

Hamilton AFDC, Jones KE, Wolpert DM (2004) The scaling of motor noise with muscle strength and motor unit number in humans. Exp Brain Res 157:417-430.

Harris CM, Wolpert DM (1998) Signal-dependent noise determines motor planning. Nature 394:780-784.

Howard IS, Ingram JN, Wolpert DM (2009) A modular planar robotic manipulandum with end-point torque control. J Neurosci Methods 181:199-211.

Izawa J, Rane T, Donchin O, Shadmehr R (2008) Motor adaptation as a process of reoptimization. J Neurosci 28:2883-2891.

Jones KE, Hamilton AF, Wolpert DM (2002) Sources of signal-dependent noise during isometric force production. J Neurophysiol 88:1533-1544.

Knill DC, Bondada A, Chhabra M (2011) Flexible, task-dependent use of sensory feedback to control hand movements. J Neurosci 31:1219-1237.

Liu D, Todorov E (2007) Evidence for the flexible sensorimotor strategies predicted by optimal feedback control. J Neurosci 27:93549368.

McIntyre J, Zago M, Berthoz A, Lacquaniti F (2001) Does the brain model Newton's laws? Nat Neurosci 4:693-694.

Milner TE, Franklin DW (2005) Impedance control and internal model use during the initial stage of adaptation to novel dynamics in humans. J Physiol 567:651-664.

Nagengast AJ, Braun DA, Wolpert DM (2009) Optimal control predicts human performance on objects with internal degrees of freedom. PLoS Comput Biol 5:e1000419.

Nashed JY, Crevecoeur F, Scott SH (2012) Influence of the behavioral goal and environmental obstacles on rapid feedback responses. J Neurophysiol 108:999-1009.

Nashed JY, Crevecoeur F, Scott SH (2014) Rapid online selection between multiple motor plans. J Neurosci 34:1769-1780.

Oldfield R (1971) The assessment and analysis of handedness: the Edinburgh inventory. Neuropsychologia 9:97-113.

Oostwoud Wijdenes L, Brenner E, Smeets JBJ (2011) Fast and finetuned corrections when the target of a hand movement is displaced. Exp Brain Res 214:453-462.

Prablanc C, Martin O (1992) Automatic control during hand reaching at undetected two-dimensional target displacements. J Neurophysiol 67:455-469.
Pruszynski JA, Scott SH (2012) Optimal feedback control and the long-latency stretch response. Exp Brain Res 218:341-359.

Pruszynski JA, Kurtzer I, Scott SH (2008) Rapid motor responses are appropriately tuned to the metrics of a visuospatial task. J Neurophysiol 100:224-238.

Qian N, Jiang Y, Jiang ZP, Mazzoni P (2013) Movement duration, Fitts's law, and an infinite-horizon optimal feedback control model for biological motor systems. Neural Comput 25:697-724.

Raftery AE, Kass RE (1995) Bayes factors. J Am Stat Assoc 90:773795.

Reichenbach A, Franklin DW, Zatka-Haas P, Diedrichsen J (2014) A dedicated binding mechanism for the visual control of movement. Curr Biol 24:780-785.

Reynolds RF, Day BL (2012) Direct visuomotor mapping for fast visually-evoked arm movements. Neuropsychologia 50:3169-3173.

Rigoux L, Guigon E (2012) A model of reward- and effort-based optimal decision making and motor control. PLoS Comput Biol 8: e1002716.

Russo AA, Khajeh R, Bittner SR, Perkins SM, Cunningham JP, Abbott LF, Churchland MM (2019) Neural trajectories in the supplementary motor area and primary motor cortex exhibit distinct geometries, compatible with different classes of computation. bioRxiv 650002. doi: 10.1101/650002.

Sarlegna F, Blouin J, Bresciani JP, Bourdin C, Vercher JL, Gauthier GM (2003) Target and hand position information in the online control of goal-directed arm movements. Exp Brain Res 151:524-535.

Saunders JA, Knill DC (2003) Humans use continuous visual feedback from the hand to control fast reaching movements. Exp Brain Res 152:341-352.

Saunders JA, Knill DC (2004) Visual feedback control of hand movements. J Neurosci 24:3223-3234.

Saunders JA, Knill DC (2005) Humans use continuous visual feedback from the hand to control both the direction and distance of pointing movements. Exp Brain Res 162:458-473.

Scheidt RA, Reinkensmeyer DJ, Conditt MA, Rymer WZ, MussaIvaldi FA (2000) Persistence of motor adaptation during constrained, multi-joint, arm movements. J Neurophysiol 84:853-862.

Schwarz G (1978) Estimating the dimension of a model. Ann Statist 6:461-464.

Shadmehr R, Huang HJ, Ahmed AA (2016) A representation of effort in decision-making and motor control. Curr Biol 26:1929-1934.

Todorov E (2005) Stochastic optimal control and estimation methods adapted to the noise characteristics of the sensorimotor system. Neural Comput 17:1084-1108.

Todorov E, Jordan MI (2002) Optimal feedback control as a theory of motor coordination. Nat Neurosci 5:1226-1235.

Todorov E, Li W (2005). A generalized iterative LQG method for locally-optimal feedback control of constrained nonlinear stochastic systems. Proceedings of the 2005, American Control Conference, Portland, OR, USA, 2005, pp 300-306.

Yeo SH, Franklin DW, Wolpert DM (2016) When optimal feedback control is not enough: feedforward strategies are required for optimal control with active sensing. PLoS Comput Biol 12:e1005190.

Zago M, Bosco G, Maffei V, Iosa M, Ivanenko YP, Lacquaniti F (2004) Internal models of target motion: expected dynamics overrides measured kinematics in timing manual interceptions. J Neurophysiol 91:1620-1634.

Zhang Y, Brenner E, Duysens J, Verschueren S, Smeets JB (2018) Postural responses to target jumps and background motion in a fast pointing task. Exp Brain Res 236:1573-1581. 